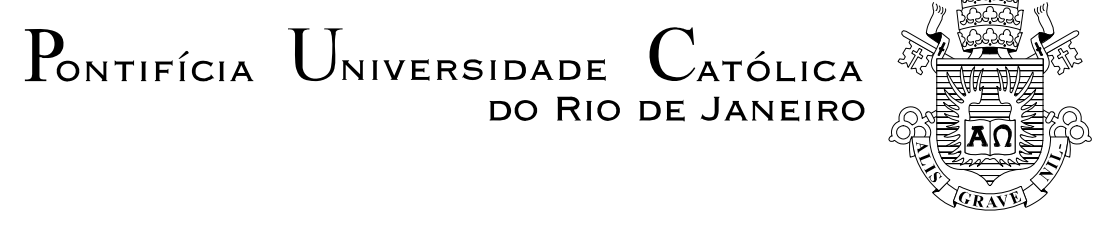

Felipe Santos Araújo

\title{
Avaliação Logística e Econômica para o Uso de Gás Natural Liquefeito em duas Plantas Industriais
}

Dissertação de Mestrado

Dissertação apresentada como requisito parcial para obtenção do título de Mestre em Logística pelo Programa de Pós-Graduação em Engenharia Industrial da PUC-Rio.

Orientador: Silvio Hamacher

Rio de Janeiro Dezembro de 2007 


\title{
Pontifícia Universidade Católica $_{\text {ata }}$ \\ DO RIO DE JANEIRO

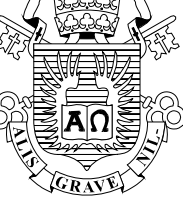

Felipe Santos Araújo

\section{Avaliação Logística e Econômica para o Uso de Gás Natural Liquefeito em duas Plantas Industriais}

\begin{abstract}
Dissertação apresentada como requisito parcial para obtenção do título de Mestre em Logística pelo Programa de Pós-Graduação em Engenharia Industrial da PUC-Rio. Aprovada pela Comissão Examinadora abaixo assinada.
\end{abstract}

Silvio Hamacher Orientador

Departamento de Engenharia Industrial -PUC-Rio

Prof. José Eugênio Leal

Departamento de Engenharia Industrial -PUC-Rio

Giovani Vitória Machado

Empresa de Pesquisa Energética - EPE

Prof. José Eugênio Leal Coordenador Setorial de Pós-Graduação do Centro Técnico Científico (PUC-Rio)

Rio de Janeiro, 12 dezembro de 2007. 
Todos os direitos reservados. É proibida a reprodução total ou parcial do trabalho sem autorização da universidade, do autor e do orientador.

Felipe Santos Araújo

Graduou-se em Engenharia Química na UERJ (Universidade do Estado do Rio de Janeiro) em 2004. Trabalhou na Esso Brasileira de Petróleo nas áreas de logística, qualidade, laboratório e implementação de sistemas ERP. Atualmente trabalha em Suprimentos na Companhia Vale do Rio Doce.

Ficha Catalográfica

Araújo, Felipe Santos

Avaliação logística e econômica para uso de gás natural liquefeito em duas plantas industriais / Felipe Santos Araújo ; orientador: Silvio Hamacher. - 2007.

133 f. : il. ; $30 \mathrm{~cm}$

Dissertação (Mestrado em Engenharia Industrial)Pontifícia Universidade Católica do Rio de Janeiro, Rio de Janeiro, 2007.

Inclui bibliografia

1. Engenharia industrial - Teses. 2. Gás Natural liquefeito. 3. Matriz energética. 4. Logística. 5. Gestão de Estoque. I. Hamacher, Silvio. II. Pontifícia Universidade Católica do Rio de Janeiro. Departamento de Engenharia Industrial. III. Título.

CDD: 658.5 


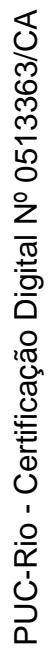

Dedico à minha família. 


\section{Agradecimentos}

Ao professor Silvio Hamacher, pela orientação, paciência, objetividade, competência e profissionalismo.

Aos meus pais e mestres Sérgio e Adelina e as minhas irmãs queridas. Agradeço por serem motivos de orgulho, meu porto seguro e fonte inesgotável de amor.

A Tatiana, minha linda. Além do maravilhoso carinho de todos os dias, agradeço pelo infinito incentivo e inspiração.

A todos os amigos e Confrades.

Aos membros da Comissão Examinadora pelas contribuições ao trabalho.

Aos mestres e colegas do Mestrado Profissional em Logística da PUC-Rio. 


\section{Resumo}

Araújo, Felipe Santos; Hamacher, Silvio. Avaliação Logística e Econômica para o Uso de Gás Natural Liquefeito em duas Plantas Industriais. Rio de Janeiro, 2007. 133p. Dissertação de Mestrado Departamento de Engenharia Industrial, Pontifícia Universidade Católica do Rio de Janeiro.

Esta dissertação apresenta dois estudos de caso de Plantas Industriais que atualmente utilizam Óleo Combustível e/ou Carvão como fontes de energia para seus processos. Dada a grande relevância destes combustíveis em seus custos de manufatura e seu caráter estratégico para a estabilidade das operações, torna-se extremamente pertinente a busca contínua por alternativas mais econômicas, com garantia de oferta no longo prazo e atendimento aos requisitos ambientais cada vez mais restritivos. Uma particularidade importante para as duas plantas é a sua localização em regiões brasileiras sem oferta de Gás Natural (GN) canalizado. Neste contexto, o Gás Natural Liquefeito (GNL) é a alternativa logística estudada neste trabalho para suprimento de GN para as plantas estudadas. Ainda não ocorrem no Brasil importações de GNL, entretanto a primeira descarga do combustível deve ocorrer em breve e pode passar a ser fonte regular de energia para diversos consumidores. Exatamente por ser tão recente enquanto alternativa de suprimento de energia para o mercado brasileiro, busca-se conhecer suas características logísticas e, considerando o valor de investimento necessário, avaliar a viabilidade econômica de sua implementação.

\section{Palavras-chave}

Gás Natural Liquefeito, Matriz Energética, Logística, Gestão de Estoque. 


\section{Abstract}

Araújo, Felipe Santos; Hamacher, Silvio. Logistical and Economical Evaluation of the Usage of Liquefied Natural Gas in two Industrial Plants. Rio de Janeiro, 2007. 133p M. Sc. Dissertation - Department of Industrial Engeneering, Pontifícia Universidade Católica do Rio de Janeiro.

This dissertation presents two case studies of Industrial Plants that currently use Fuel Oil and/or Coal as source of energy for its processes. Given the great importance of these fuels in the manufacturing costs and its strategic role to the stability of the operations, it is extremely relevant the continuous search for most economic alternatives, with long term supply security and alignment with more and more restrict environmental standards. An important particularity of these two plants is its location in Brazilian areas without availability of Natural Gas (NG) in pipelines. Therefore, the Liquefied Natural Gas (LNG) is the logistic alternative to supply the NG to the plants under analysis. Still Brazil does not import LNG, however the first unloading of this fuel shall happen very soon and may become a regular source of energy to many consumers. Exactly for being such a recent energy supply alternative to the Brazilian market, it is so important to understand its logistic and, considering the value of needed investments, evaluate its economic viability of implementation.

\section{Keywords}

Liquefied Natural Gas, Energy Matrix, Logistic, Stock Management. 


\section{Sumário}

1. INTRODUÇÃO 15

1.1. OBJETIVOS DA PESQUISA 16

1.2. DELIMITAÇÕES DO ESCOPO DA DISSERTAÇÃO 16

1.3. ESTRUTURA DA DISSERTAÇÃO 17

2. REVISÃO BIBLIOGRÁFICA 19

2.1. DEFINIÇÕES 19

2.2. ENERGIA NO MUNDO 20

2.3. GÁS NATURAL NO MUNDO 23

2.3.1. Mercado 21

2.3.2. Produção 29

2.3.3. Transporte 30

2.4. GÁS NATURAL NO BRASIL 40

2.5. ÓLEO COMBUSTÍVEL 44

2.6. ESTUDOS DE CASO 45

2.6.1. LNG como estratégia para desenvolvimento do mercado de gás 46

2.6.2. Planta de liquefação na Califórnia 46

2.6.3. Impacto ambiental na substituição do OC pelo GN em Paulínia 47

2.6.4. Mercado nordestino de GN 47

2.6.5. Fatores condicionantes ao desenvolvimento de projeto de GNL para o Cone Sul $\quad 47$

3. METODOLOGIA 49

3.1. DEFINIÇÃO DE ESTUDO DE CASO 50

3.2. FORMULAÇÃO DO PROBLEMA 50 
3.4. ANÁLISE DE DADOS 53

3.5. ESTUDO DAS CONFIGURAÇÕES DE REFERÊNCIA 54

3.6. ESTUDO DAS CONFIGURAÇÕES PROPOSTAS 55

4. CONFIGURAÇÃO DE REFERÊNCIA 56

4.1. CONFIGURAÇÃO DE REFERÊNCIA PARA PLANTA 156

4.1.1. Logística do Óleo Combustível 57

4.1.2. Logística do carvão 59

4.2. CONFIGURAÇÃO DE REFERÊNCIA PARA PLANTA 261 4.2.1. Logística do Óleo Combustível 62

4.3. CUSTOS DAS SOLUÇÕES ATUAIS 64

5. CONFIGURAÇÃO PROPOSTA 66

5.1. CONFIGURAÇÃO PROPOSTA PARA PLANTA 1

5.2. CONFIGURAÇÃO PROPOSTA PARA PLANTA 2

5.3. CONFIGURAÇÃO DE LOGÍSTICA INTEGRADA PARA AS PLANTAS 1E 2

5.4. CUSTOS DAS CONFIGURAÇÕES PROPOSTAS 79

6. DISCUSSÃO DE DADOS 82

6.1. ANÁLISE DE INVESTIMENTO 84

6.2. ANÁLISE DE SENSIBILIDADE 85

6.2.1. Critério para Escolha das Variáveis 86

6.2.2. Critério para Variação dos Valores 87

6.2.3. Resultados das análises de sensibilidade 87

6.2.3.1 Investimento na Planta de Regaseificação 90 
6.2.3.2 Demanda de Óleo Combustível $\quad 92$

6.2.3.3 Tamanho de Lote de GNL 94

6.2.3.4 Taxa de Câmbio (R\$ / USD) 97

6.2.3.5 Preço do GNL 98

6.2.3.6 Preço do OC e Carvão 102

6.2.3.7 Custo de Capital $\quad 105$

$\begin{array}{lll}\text { 7. CONCLUSÃO } & 107\end{array}$

7.1. RECOMENDAÇÕES PARA TRABALHOS FUTUROS 109

8. REFERÊNCIAS BIBLIOGRÁFICAS

APÊNDICE I - MEMÓRIA DE CÁLCULO DA PLANTA 1

I.1. SOLUÇÃO ATUAL - CUSTO COM OC 116

1.2. SOLUÇÃO ATUAL - CUSTO COM CARVÃO 118

1.3. SOLUÇÃO ATUAL - CUSTO TOTAL 120

l.4. SOLUÇÃO PROPOSTA - CUSTO COM GNL 121

I.5. SOLUÇÃO PROPOSTA - INVESTIMENTO E ANÁLISE 123

I.6. ANÁLISE DE SENSIBILIDADE 124

APÊNDICE II - MEMÓRIA DE CÁLCULO DA PLANTA 2

II.1. SOLUÇÃO ATUAL - CUSTO COM OC 126

II.2. SOLUÇÃO ATUAL - CUSTO TOTAL 128

II.3. SOLUÇÃO PROPOSTA - CUSTO COM GNL 129

II.4. SOLUÇÃO PROPOSTA - INVESTIMENTO E ANÁLISE 130

II.5. ANÁLISE DE SENSIBILIDADE 131

APÊNDICE III - SOLUÇÃO PROPOSTA COM LOGÍSTICA 


\section{Lista de Figuras}

Figura 2.1 - Previsão de Matriz Enérgica Mundial até 2030

Figura 2.2 - Importações de GNL por Continente 25

Figura 2.3 - Exportações de GNL por Continente 25

Figura 2.4 - Comparação de Custos para transporte de GN 31

Figura 2.5 - Cadeia de Suprimento do GNL 34

Figura 2.6 - Processo de Liquefação do Gás Natural 36

Figura 2.7 - Processo de Regaseificação do GNL 39

Figura 2.8 - Matriz Energética Brasileira 40

Figura 2.9 - Evolução da Importação de Gás Natural no Brasil 41

Figura 2.10 - Esquema geral de Refino de Petróleo 45

Figura 4.1 - Macrofluxo da Cadeia de Suprimento de OC para

Planta $1 \quad 58$

Figura 4.2 - Macrofluxo da Cadeia de Suprimento de Carvão para Planta $1 \quad 61$

Figura 4.3 - Macrofluxo Cadeia de Suprimento de OC para Planta 263

Figura 5.1 - Prováveis Rotas de Combustíveis para Plantas 1 e $2 \quad 68$

Figura 5.2 - Planta e Terminal de Liquefação Atlantic LNG 69

Figura 5.3 - Macrofluxo Cadeia de Suprimento de GNL para Planta 170

Figura 5.4 - Layout Planta 1

Figura 5.5 - Macrofluxo Cadeia de Suprimento de GNL para Planta 275

Figura 5.6 - Layout Planta $2 \quad 76$

Figura 5.7 - Cenários de Logística de GNL integrada para Plantas 1 e 2 78

Figura 6.1.a - Abertura de custos por combustível - Planta 1

Figura 6.1.b - Abertura de custos por combustível - Planta 2

Figura 6.2.a - Abertura de custos por combustível - Planta 1

Figura 6.2.b - Abertura de custos por combustível - Planta $2 \quad 84$

Figura 6.3 - Sumário de Análise de Sensibilidade por

Payback Descontado

Figura 6.4 - Sumário de Análise de Sensibilidade por Taxa de Retorno em 10 anos

Figura 6.5.a - Análise de Sensibilidade sobre Investimento na Planta 
de Regaseificação - Planta 1

Figura 6.5.b - Análise de Sensibilidade sobre Investimento na Planta de Regaseificação - Planta 2

Figura 6.6.a - Análise de Sensibilidade sobre Demanda de Óleo Combustível - Planta 1

Figura 6.6.b - Análise de Sensibilidade sobre Demanda de Óleo Combustível - Planta 1

Figura 6.6.c - Análise de Sensibilidade sobre Demanda de Óleo Combustível - Planta 2

Figura 6.6.d - Análise de Sensibilidade sobre Demanda de Óleo Combustível - Planta 2

Figura 6.7.a - Análise de Sensibilidade sobre Tamanho de Lote de GNL - Planta 1

Figura 6.7.b - Análise de Sensibilidade sobre Tamanho de Lote de GNL - Planta 1

Figura 6.7.c - Análise de Sensibilidade sobre Tamanho de Lote de GNL - Planta 2

Figura 6.7.d - Análise de Sensibilidade sobre Tamanho de Lote de GNL - Planta 2 96

Figura 6.8.a - Análise de Sensibilidade sobre Câmbio - Planta 1 97

Figura 6.8.b - Análise de Sensibilidade sobre Câmbio - Planta 1 97 Figura 6.9 - Preços spot de GNL 99 Figura 6.10.a - Análise de Sensibilidade sobre Preço do GNL Planta 1 100

Figura 6.10.b - Análise de Sensibilidade sobre Preço do GNL Planta 1

Figura 6.10.c - Análise de Sensibilidade sobre Preço do GNL Planta 2

Figura 6.10.d - Análise de Sensibilidade sobre Preço do GNL Planta 2

Figura 6.11.a - Análise de Sensibilidade sobre Preço do OC e Carvão - Planta 1

Figura 6.11.b - Análise de Sensibilidade sobre Preço do OC e Carvão - Planta 1 
Figura 6.11.c - Análise de Sensibilidade sobre Preço do OC e Carvão - Planta 1

Figura 6.11.d - Análise de Sensibilidade sobre Preço do OC e Carvão - Planta 1

Figura 6.11.e - Análise de Sensibilidade sobre Preço do OC e Carvão - Planta 2

Figura 6.11.f - Análise de Sensibilidade sobre Preço do OC e Carvão - Planta 2

Figura 6.12.a - Análise de Sensibilidade sobre Custo de Capital Planta 1

Figura 6.12.b - Análise de Sensibilidade sobre Custo de Capital Planta 2 


\section{Lista de Tabelas}

Tabela 2.1 - Demanda de GNL por Continente, Capacidade de Liquefação e Transporte

Tabela 2.2 - Demanda GNL, Capacidade Liquefação e Capacidade de Transporte

Tabela 2.3 - Demanda de GNL por país 27

Tabela 2.4 - Resumo de Capacidade Global de Liquefação 27

Tabela 2.5 - Países Produtores de GNL para mercado do Atlântico $\quad 27$

Tabela 2.6 - Produção de GN por Unidade Federativa no Brasil $\quad 41$

Tabela 2.7 - Consumo de GN no Brasil por segmento 42

Tabela 3.1 - Demanda Fonte de Dados 53

Tabela 4.1 - Custos com Fornecimento de Combustíveis para

Planta 1

Tabela 4.2 - Custos com Fornecimento de Combustíveis para

Planta 2

64

Tabela 5.1 - Capacidade Global de Liquefação $\quad 67$

Tabela 5.2 - Cenários de Logística de GNL integrada para Plantas 1 e 2

Tabela 5.3 - Custos com Fornecimento de GNL para Planta $1 \quad 79$

Tabela 5.4 - Custos com Fornecimento de GNL para Planta $2 \quad 80$

Tabela 5.5 - Custos com Fornecimento de GNL para Planta 1 com integração logística

Tabela 5.6 - Custos com Fornecimento de GNL para Planta 2 com integração logística 80

Tabela 6.1 - Análise de Investimento 85

Tabela 6.2 - Análise de Sensibilidade - Planta $1 \quad 88$

Tabela 6.3 - Análise de Sensibilidade - Planta $2 \quad 89$ 